Public Health

Original Research Study

Central Eur J Paed 2021;17(1):27-39

DOI: $10.5457 / \mathrm{p} 2005-114.284$

\title{
Mental and Physical Health of Students Who Study in Post-Conflict North Kosovo
}

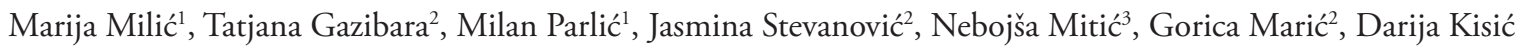 \\ Tepavčević ${ }^{2}$ Tatjana Pekmezović \\ ${ }^{1}$ Department of Epidemiology, Faculty of Medicine, University of Priština temporarily settled in Kosovska Mitrovica, Kosovska \\ Mitrovica, Kosovo, Serbia, ${ }^{2}$ Institute of Epidemiology, Faculty of Medicine, University of Belgrade, Belgrade, Serbia, ${ }^{3}$ Institute \\ of Pathophysiology, Faculty of Medicine, University of Priština temporarily settled in Kosovska Mitrovica, Kosovo, Serbia
}

Correspondence: pekmezovic@sezampro.rs; Tel. / Fax.: + 381113607062

Received: December 31, 2020; Accepted: February 25, 2021

\begin{abstract}
Objective - To examine health-related quality of life (HRQoL) among Serbian students who study in the northern Kosovo province and to explore potential differences in HRQoL relative to students' region of origin. Materials and Methods - This study was conducted from April to June 2015. A total of 514 students from the University of Priština temporarily settled in Kosovska Mitrovica were enrolled in the study. The students completed the socio-demographic and lifestyle questionnaire, Beck Depression Inventory (BDI) and the generic HRQoL questionnaire Short Form-36 (SF-36). The region of origin was classified based on the area in which the students were originally from: Republic of Serbia vs. North Kosovo vs. Southern enclaves. Results - The highest scores were observed for Physical Functioning and the lowest for Vitality. No difference in HRQoL was observed between students from the Republic of Serbia, North Kosovo and Southern enclaves. Compared to females, males scored better on Bodily Pain, General Health, Social Functioning and Mental Health. They also had better Physical and Mental Composite scores and Total HRQoL than females. Worse depressive symptoms were consistently associated with poorer HRQoL across all three groups of students. Conclusion - The HRQoL among Serbian students in the northern Kosovo province does not differ based on their region of origin. Health care services for university students should prioritize prevention, early recognition and treatment of depressive symptoms.
\end{abstract}

Key Words: Students - University - Health-Related Quality Of Life • Post-Conflict.

\section{Introduction}

The value of years of life spent in good health has become increasingly relevant because of the global ageing and increase in life expectancy (1). At university, students take on responsibility for own education as well as for own health and health-related behaviors. At the same time, they may be exposed to numerous stressors related to the academic environment, such as school achievements, peer competition, fear of failing (2), adaptation to the new living circumstances, building new social circles and financial constraints (3). This may explain pre- vious evidence that university students have poorer health-related quality of life (HRQoL) compared to community-based population of the same age $(4,5)$.

Previous studies suggested that HRQoL of university students is influenced by the type of school program, age, gender, place of residence, level of physical activity, quality of social support, academic performance, health status, finances and mental health challenges, such as depression, anxiety and burnout (6-9). Bearing in mind that university students will have the leading role in the development of education, economy, law, health and culture in 
the near future, this population group represents an important component of the social capital in the community. Thus, it is essential to recognize their health challenges early on.

The territory of Kosovo and Metohija has been disputed between the Albanians and Serbs, which resulted in an armed conflict in 1999. Following this, Kosovo and Metohija was administered by the United Nations Mission in Kosovo (UNMIK, UN Security Council Resolution 1244) (10). In 2008, the Albanian majority in Kosovo unanimously declared independence. As a result, most Serbs living in Kosovo fled to the north of Kosovo (specifically to 4 municipalities: Kosovska Mitrovica, Zvečan, Zubin Potok and Leposavić), while some Serbs remain in the enclaves (settlements) in the southern Kosovo (Fig. 1) (11).

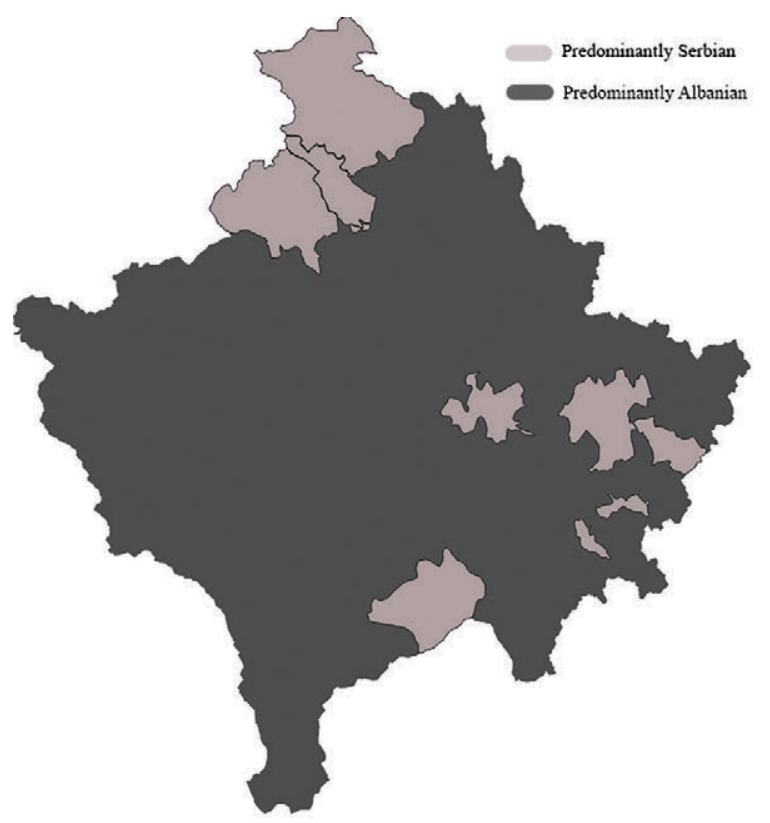

Fig. 1. Map of Kosovo according to Ethnic Groups

The Serbs in Kosovo are currently being considered as an ethnic minority. According to the Constitution of the Republic of Serbia, the province of Kosovo and Metohija is an integral part of the Republic of Serbia (12). In North Kosovo, the Kosovo Government administration is not fully operational. However, in the southern enclaves this is not the case. Due to the previous conflict, Serbian municipalities both in North Kosovo and in the southern enclaves, are underdeveloped and rates of unemployment are high. People who live in these areas have reduced mobility, particularly in the enclaves (13).

Given the challenges of the academic environment, we assumed that students in North Kosovo might be exposed to greater stressors (due to political instability and safety issues) compared to their peers who live and study in other regions $(14,15)$. The classes at the university in North Kosovo are organized in such a way that weekly commutes between the campus and their home towns are common. So, students do not entirely leave their home environment after having been enrolled in the university. Because levels of mobility, economic potential and social set-up differ between regions, we hypothesized that students who came to the university from the Republic of Serbia, North Kosovo and southern enclaves might perceive their HRQoL as different. This was a unique opportunity to explore HRQoL of people of the same ethnic background, who speak the same language, but come from different geo-political settings.

The aim of this study was to assess health-related quality of life among students who study in the northern Kosovo province and examine potential differences in HRQoL relative to students' region of origin.

\section{Materials and Methods}

\section{Setting}

This study was conducted at the University of Priština temporarily settled in Kosovska Mitrovica. Priština is the capital city of the Kosovo province (16). The University of Priština was founded 1969, however, after the conflict in 1999, the University was relocated to Serbia (from 1999 to 2001). Two years later (2003), the seat of the University of Priština was transferred to Kosovska Mitrovica (North Kosovo) (16). The city of Kosovska Mitrovica is divided by the river Ibar in two parts: the northern part, predominantly settled by the Serbs (approximately 23,000 inhabitants), and the south- 
ern part, predominantly settled by the Albanians (approximately 50,000 inhabitants).

The University of Priština temporarily settled in Kosovska Mitrovica is located in the northern part of the city. The classes are taught in the Serbian language. The University consists of 10 schools (social sciences and humanities, medical sciences, nature sciences and mathematics, and technology and engineering sciences) with approximately 8,000 students (16). Students at the University come from North Kosovo, southern enclaves as well as from the Republic of Serbia.

\section{Participants}

This study was designed as a cross-sectional study. Participants were the students from the University of Priština temporarily settled in Kosovska Mitrovica, who came for mandatory health checks at the Student Health Care Center in Kosovska Mitrovica. The survey was conducted from April to June, 2015. The minimum sample size calculated was 367 using online calculator at http://www.raosoft.com/samplesize.html (based on the following parameters: population $-8,000$; response distribution $-50 \%$ : confidence interval $-95 \%$ and margin of error $5 \%$ ). A total of 514 consecutive students were included. The number of the recruited students represents approximately $6.4 \%$ of all students at the University. Participation was voluntary and anonymous. Students provided oral informed consent.

\section{Instruments}

The participants completed a questionnaire about socio-demographics (gender, age, type of faculty [social sciences and humanities/health sciences/ natural sciences and mathematics/technology and engineering sciences], household monthly income, region of birth and current living arrangement [living at home with parents/living in dormitory/ living alone in a rented apartment/other living arrangements such as living with relatives, in a bedand-board type of accommodation or in a hotel]) health behaviors (smoking, alcohol use, physical activity) and health status (having chronic diseases). Smokers were defined as persons who reported everyday smoking during a 60-day period. Alcohol consumption was determined by the frequency of drinking as follows: everyday, weekly, occasionally and never. To assess the practice of physical activity, students were asked whether they engaged in activities for at least $10 \mathrm{~min}$ at a time, such as brisk walking, cycling, swimming, or any other activity that causes some increase in breathing or heart rate. A chronic disease was considered as an illness which requires medical treatment or limits daily activities for prolonged period of time e.g 1 year or more. Students were provided a list of most common chronic diseases in a university student population and an additional space to add a non-listed disease. These data were compiled into one variable with binary answer yes vs. no.

The HRQoL was assessed using the SF-36 questionnaire (Serbian translation) (17). The SF36 comprises 36 questions which examine the eight dimensions of health: Physical Functioning (PF), Role Functioning Physical (RP), Bodily Pain (BP), General Health (GH), Vitality (VT), Social Functioning (SF), Role Functioning Emotional (RE), and Mental Health (MH). The former four domains comprise the Physical Composite score (PCS) and the latter four domains comprise the Mental Composite score (MCS). The scores range from 0 to 100 , with higher scores suggesting better self-perceived health.

The Beck Depression Inventory (BDI) evaluates the presence of depressive symptoms (18). The scale is composed of 21 items. The answers are ranked on a scale from 0 to 3 . The rank 0 denotes no symptoms and the rank 3 denoted intense symptoms. The BDI score represents the sum of item scores, with higher scores indicating more severe depressive symptoms (18).

\section{Ethics Statement}

The Ethics Committee of the Faculty of Medicine, University of Priština temporarily seated in Kosovska Mitrovica approved the study (approval no. 01503; issued on April 2, 2015). 


\section{Statistical Analyses}

Data were analyzed using the SPSS for Windows, version 20.0. We observed that variables 'household monthly income' and 'region of origin' had $>20 \%$ missing data. To reduce potential bias from missing data and to increase precision, we performed the multiple imputation of 'household monthly income' and 'region of origin'. We generated 20 independent imputed datasets using the Markov Chain Monte Carlo method and calculated pooled estimates (19). We stratified the study sample according to basic demographic characteristics, such as gender (male vs. female), living arrangements (home vs. dormitory vs. rented apartment vs. other) and type of faculty (Social sciences and humanities vs. Health sciences vs. Natural sciences and mathematics vs. Technology and engineering sciences). Differences between the HRQoL scores were examined using the ANOVA. The post hoc Bonferroni correction was used to find the exact differences between multiple groups (for variable 'living arrangements' and 'type of faculty'). The Spearman's correlation coefficient was used to examine the correlation.

We used the digital acyclic graphs (DAG) to visualize potential associations between the variables. The DAGs were created using an open-source platform www.dagitty.net. A previous study found that depression was the strongest contributor to poorer HRQoL among university students (20). For this reason, in the final DAG we considered "depression" (as measured by the BDI) as the exposure and "HRQoL" as the outcome. We observed that the 'region of origin' might be a moderator of the association between BDI and HRQoL (red circle in DAG), while other variables were deemed as mediators (blue circles in DAG) (Supplemental Fig. $\mathrm{S} 1$ ). Based on the DAG, we ran a multiple linear regression model stratified according to region of origin (Serbia/North Kosovo/Southern enclaves). The dependent variable was the total HRQoL score as measured by the PCS and MCS. The independent variables were: age, gender, living arrangements, household monthly income, type of school, grade point average, smoking, alcohol intake, physical activity, having chronic diseases and BDI. Testing of multicollinearity showed that none of the independent variables had a variance inflation factor of >2.0. Therefore, no variable was excluded. The effect estimates were presented as beta coefficients, with corresponding 95\% confidence intervals. The probability level $(\mathrm{P})$ was set at 0.05 . Estimates were presented for both the non-imputed and the imputed datasets.

\section{Results}

\section{Description of the Study Sample}

The demographic characteristics of study participants are presented in Table 1. Of 514 students, 238 (46.3\%) were male and $276(53.7 \%)$ were female. The average age of students was $20.8 \pm 1.6$ years. Almost one-half of the participants (46.3\%) were students in health sciences. Most students (36.8\%) reported living in dormitories. The largest proportion of students from the University of Priština, temporarily seated in Kosovska Mitrovica, were from Serbia (31.9\%), 16.1\% were from North Kosovo and $13.4 \%$ were from the southern enclaves (non-imputed dataset). Considerable proportion of students did not report their region of origin (38.5\%).

\section{Health-Related Quality of Life Scores}

The mean scores of SF-36 according region of origin are presented in Table 2. The highest scores in the non-imputed dataset were observed for Physical Functioning (student from Serbia 95.4, students from North Kosovo 92.4 and students from enclaves 93.7, $\mathrm{P}=0.489)$. The lowest were observed for Vitality (student from Serbia 68.8, students from North Kosovo 73.6 and students from enclaves 68.0, $\mathrm{P}=0.208)$. In the non-imputed dataset, students from southern enclaves scored significantly poorer on Body Pain domain compared to students from Serbia and North Kosovo (students from enclaves 82.3 vs. students from Serbia 85.8 vs. students from North Kosovo 89.7; $\mathrm{P}=0.024)$. 
Table 1. Socio-Demographic Characteristics of Students at the University of Priština Temporarily Seated at

Kosovska Mitrovica ( $\mathrm{N}=503)$

\begin{tabular}{|c|c|c|}
\hline Variable & $\mathrm{N}(\%)$ & \\
\hline Median age in years (IR) & $21.0(2.0)$ & \\
\hline \multicolumn{3}{|l|}{ Gender } \\
\hline Male & $238(46.3)$ & \\
\hline Female & $276(53.7)$ & \\
\hline \multicolumn{3}{|l|}{ Current living arrangement } \\
\hline Home (with parents) & $140(27.2)$ & \\
\hline Students' dormitory & $189(36.8)$ & \\
\hline Alone (in rented apartment) & $170(33.1)$ & \\
\hline $\begin{array}{l}\text { Other (with relatives, bed-and- } \\
\text { board, hotel) }\end{array}$ & $15(2.9)$ & \\
\hline \multicolumn{3}{|l|}{ Type of school } \\
\hline Social sciences and humanities & $143(27.8)$ & \\
\hline Health sciences & $238(46.3)$ & \\
\hline Natural sciences and mathematics & $14(2.7)$ & \\
\hline Technology and engineering sciences & $119(23.2)$ & \\
\hline Median grade point average (IR) & $7.8(1.25)$ & \\
\hline Smoking & $111(22.1)$ & \\
\hline Alcohol intake & $342(68.0)$ & \\
\hline Physical activity & 407 (80.9) & \\
\hline Having chronic diseases & $53(10.5)$ & \\
\hline \multicolumn{3}{|l|}{ Imputed } \\
\hline Region of origin & Observed & Imputed \\
\hline Serbia & $164(31.9)$ & $269(53.5)$ \\
\hline North of Kosovo & $83(16.1)$ & $124(24.7)$ \\
\hline Southern enclaves & $69(13.4)$ & $110(21.9)$ \\
\hline Missing & $198(38.5)$ & - \\
\hline \multicolumn{3}{|l|}{ Household monthly income } \\
\hline Quartile 1 & $81(16.1)$ & $130(25.8)$ \\
\hline Quartile 2 & $92(18.3)$ & $141(28.0)$ \\
\hline Quartile 3 & $67(13.3)$ & $114(22.7)$ \\
\hline Quartile 4 & $72(14.3)$ & $118(23.5)$ \\
\hline Missing & $191(38.0)$ & - \\
\hline \multirow{2}{*}{ Median Beck Depression Inventory score } & $\mathrm{N}=476$ & $\mathrm{~N}=503$ \\
\hline & $1.0(5.0)$ & $1.0(5.0)$ \\
\hline
\end{tabular}

The HRQoL scores according to gender are presented in Supplemental Table S1. Males scored significantly better on Bodily Pain (males 88.0 vs. females 83.8, $\mathrm{P}=0.002$ ), General Health (males 77.1 vs. females 72.0, $\mathrm{P}=0.001$ ), Social Functioning (males 83.3 vs. females 79.6, $\mathrm{P}=0.014$ ) and Mental Health (males 76.3 vs. females $72.4, \mathrm{P}=0.022$ ). They also scored better on both composite scores (PCS males 82.9 vs. females 80.4, $\mathrm{P}=0.016$; MCS males 77.8 vs. 73.7 females $\mathrm{P}=0.005$ ) and the total SF-36 score compared to females (males 82.0 vs. females 78.9, $\mathrm{P}=0.007$ ).

The HRQoL scores according to living arrangements are presented in Supplemental Table S2. Students who lived "at home with parents" had better Role Physical (90.0 vs, 82.1, P=0.002), Vitality (71.0 vs. $65.9, \mathrm{P}=0.009)$, and PCS (83.8 vs. 79.3 , $\mathrm{P}=0.001)$ compared to students who lived alone. In a similar vein, students who lived with their parents scored better on Role Physical (90.4 vs. 71.7, $\mathrm{P}=0.002)$, Bodily Pain (88.2 vs. $73.5, \mathrm{P}=0.002)$, General Health (76.2 vs. 62.0, $\mathrm{P}=0.008)$, PCS (83.8 vs. $70.9, \mathrm{P}=0.001)$, $\mathrm{MCS}$ (77.4 vs. $64.7, \mathrm{P}=0.028)$ and Total HRQoL score (82.4 vs. 69.7, $\mathrm{P}=0.007$ ) compared to students who had "other" living arrangements. Students who lived in dormitories had less Bodily Pain (87.4 vs. 73.5, $\mathrm{P}=0.002$ ), better General health (75.6 vs. $62.0, \mathrm{P}=0.008)$ and PCS (82.7 vs. 70.9, $\mathrm{P}=0.001$ ) compared to students who had "other" living arrangements.

The HRQoL scores according to type of faculty are presented in Supplemental Table S3. Students who studied Health sciences had more Bodily Pain (83.6 vs. $89.6, P=0.025)$ and worse General Health (72.8 vs. $78.9 \mathrm{P}=0.006)$, Vitality (65.1 vs. 74.2, $\mathrm{P}=0.001)$ and Mental Health (72.2 vs. 79.6, $\mathrm{P}=0.001)$ compared to Technology and engineering students. Students in Health sciences reported less Vitality (65.1 vs. 70.1, $\mathrm{P}=0.001$ ) compared to students in Social sciences and humanities. Students in Social sciences had lower General Health (73.6 vs. 78.9, $\mathrm{P}=0.006)$ and Mental Health $(72.2$ vs. 79.6, $\mathrm{P}=0.001)$ compared to students in Technology and engineering (Supplemental Table S3).

The average score on BDI was 3.94 \pm 6.28 (range from 0 to 40). Statistically significant difference in BDI score was observed between male and female students $(3.5 \pm 6.5$ vs. $4.3 \pm 6.1, \mathrm{P}=0.035$, respectively). There was no correlation between the total SF-36 score and the average monthly income 
Table 2. Mean Scores of the SF-36 Scales according to Region of Origin

\begin{tabular}{|c|c|c|c|c|}
\hline \multirow{2}{*}{ Scales of SF-36 } & Serbia & North of Kosovo & Enclaves & \multirow{2}{*}{$P$ value } \\
\hline & Mean \pm SD & Mean \pm SD & Mean \pm SD & \\
\hline \multicolumn{5}{|l|}{ Non-imputed dataset } \\
\hline Physical functioning & $95.4 \pm 11.3$ & $92.4 \pm 21.3$ & $93.7 \pm 14.2$ & 0.482 \\
\hline Role physical & $87.3 \pm 23.7$ & $88.9 \pm 24.1$ & $87.7 \pm 24.9$ & 0.608 \\
\hline Pain & $85.8 \pm 17.2$ & $89.7 \pm 16.3$ & $82.3 \pm 20.1$ & $0.024^{*}$ \\
\hline General health & $75.1 \pm 17.5$ & $77.7 \pm 15.0$ & $73.4 \pm 17.6$ & 0.316 \\
\hline Vitality & $68.8 \pm 20.5$ & $73.6 \pm 19.1$ & $68.0 \pm 22.6$ & 0.208 \\
\hline Social functioning & $83.3 \pm 19.8$ & $83.4 \pm 21.6$ & $81.7 \pm 21.1$ & 0.628 \\
\hline Role emotional & $79.3 \pm 35.3$ & $82.3 \pm 33.1$ & $78.3 \pm 36.5$ & 0.753 \\
\hline Mental health & $75.8 \pm 19.1$ & $76.2 \pm 20.7$ & $72.9 \pm 22.6$ & 0.693 \\
\hline Physical composite score & $82.5 \pm 13.4$ & $84.4 \pm 12.3$ & $81.0 \pm 14.0$ & 0.176 \\
\hline Mental composite score & $76.5 \pm 16.9$ & $78.6 \pm 16.9$ & $74.9 \pm 18.5$ & 0.317 \\
\hline Total score & $81.3 \pm 14.7$ & $83.0 \pm 14.5$ & $79.7 \pm 15.9$ & 0.256 \\
\hline \multicolumn{5}{|l|}{ Imputed dataset } \\
\hline Physical functioning & $93.4 \pm 15.1$ & $90.7 \pm 22.7$ & $94.4 \pm 14.9$ & 0.215 \\
\hline Role physical & $85.8 \pm 26.1$ & $86.3 \pm 26.0$ & $85.9 \pm 26.3$ & 0.984 \\
\hline Pain & $85.2 \pm 17.5$ & $88.1 \pm 17.5$ & $83.8 \pm 18.9$ & 0.161 \\
\hline General health & $74.1 \pm 17.7$ & $75.6 \pm 16.0$ & $72.8 \pm 16.3$ & 0.446 \\
\hline Vitality & $67.5 \pm 21.2$ & $70.8 \pm 18.4$ & $68.6 \pm 20.3$ & 0.325 \\
\hline Social functioning & $81.0 \pm 22.9$ & $81.7 \pm 21.6$ & $80.7 \pm 20.6$ & 0.927 \\
\hline Role emotional & $78.7 \pm 36.0$ & $80.4 \pm 33.2$ & $78.8 \pm 36.0$ & 0.902 \\
\hline Mental health & $73.9 \pm 19.7$ & $75.2 \pm 19.4$ & $73.2 \pm 21.1$ & 0.735 \\
\hline Physical composite score & $81.2 \pm 14.1$ & $82.3 \pm 13.4$ & $81.1 \pm 13.7$ & 0.730 \\
\hline Mental composite score & $75.1 \pm 17.8$ & $76.7 \pm 16.5$ & $74.8 \pm 17.4$ & 0.614 \\
\hline Total score & $80.0 \pm 15.7$ & $81.1 \pm 14.8$ & $79.7 \pm 14.9$ & 0.746 \\
\hline
\end{tabular}

$\mathrm{SD}=$ Standard deviation; $\mathrm{P}=$ Value for ANOVA; "Significantly lower among students from enclaves vs. students from Serbia and North Kosovo.

$(\mathrm{P}=0.290)$. All SF-36 health dimensions significantly $(\mathrm{P}=0.001)$ negatively correlated with BDI.

\section{Regression Analysis}

Results of the multiple linear regression for the nonimputed and the imputed datasets are shown in Tables 3 and 4. In both the non-imputed (students from Serbia $B=-1.00,95 \%$ confidence interval $[\mathrm{CI}]:-1.59,-0.41, \mathrm{P}=0.001$; students from North Kosovo $\mathrm{B}=-1.54,95 \% \mathrm{CI}:-2.36,-0.73, \mathrm{P}=0.001$; students from southern enclaves $\mathrm{B}=-1.17,95 \% \mathrm{CI}$ : $-2.22,-0.11, \mathrm{P}=0.032$ ) and the imputed datasets (students from Serbia $B=-1.05,95 \%$ CI: -1.44 ,
-0.66, $\mathrm{P}=0.001$ : students from North Kosovo $\mathrm{B}=$ $-1.35,95 \% \mathrm{CI}$ : $-1.99,-0.71, \mathrm{P}=0.001$; students from southern enclaves $\mathrm{B}=-1.45,95 \% \mathrm{CI}:-2.57$, -0.33, $\mathrm{P}=0.012$ ) worse BDI score was associated with poorer PCS (Table 3 ).

Similarly, in all three groups of students, worse BDI was associated with poorer MCS across the two datasets (non-imputed dataset: students from Serbia $\mathrm{B}=-1.47,95 \% \mathrm{CI}:-2.03,-0.91, \mathrm{P}=0.001$; students from North Kosovo $\mathrm{B}=-2.30,95 \% \mathrm{CI}$ : $-3.30,-1.29, \mathrm{P}=0.001$; students from southern enclaves $\mathrm{B}=-2.65,95 \% \mathrm{CI}:-4.12,-1.17, \mathrm{P}=0.032$; imputed dataset: students from Serbia $B=-1.59,95 \%$ CI: $-2.07,-1.11, P=0.001$; students from North 


\begin{tabular}{|c|c|c|c|c|c|c|}
\hline \multirow{3}{*}{ Variable } & \multicolumn{6}{|l|}{ Region of origin } \\
\hline & \multicolumn{2}{|l|}{ Serbia } & \multicolumn{2}{|l|}{ North Kosovo } & \multicolumn{2}{|l|}{ Southern enclaves } \\
\hline & $\mathrm{B}(95 \% \mathrm{CI})$ & $\mathrm{P}$ & $\mathrm{B}(95 \% \mathrm{CI})$ & $\mathrm{P}$ & B $(95 \% \mathrm{CI})$ & $\mathrm{P}$ \\
\hline \multicolumn{7}{|l|}{ Non-imputed set } \\
\hline Age & $1.03(-0.48,2.55)$ & 0.178 & $-2.40(-5.19,0.39)$ & 0.088 & $-0.12(-3.80,3.55)$ & 0.945 \\
\hline Gender (male vs. female) & $0.82(-4.88,6.52)$ & 0.775 & $1.88(-6.33,10.10)$ & 0.640 & $-9.96(-17.64,-2.28)$ & 0.013 \\
\hline $\begin{array}{l}\text { Living arrangements } \\
\text { (dormitory vs. other) }\end{array}$ & $-0.03(-6.01,6.02)$ & 0.992 & $3.27(-9.88,16.44)$ & 0.612 & $-5.79(-12.40,0.82)$ & 0.083 \\
\hline Household monthly income & $0.61(-2.23,3.45)$ & 0.670 & $-0.59(-4.26,3.08)$ & 0.743 & $0.53(-3.91,4.96)$ & 0.807 \\
\hline Type of school (medicine vs. other) & $-6.47(-12.98,0.2)$ & 0.051 & $4.626(-4.60,13.85)$ & 0.311 & $-0.53(-7.56,6.50)$ & 0.877 \\
\hline Grade point average & $2.28(-0.90,5.46)$ & 0.157 & $-0.42(-5.12,4.29)$ & 0.857 & $-1.83(-9.44,5.78)$ & 0.623 \\
\hline Smoking (yes vs. no) & $-3.73(-11.26,3.80)$ & 0.326 & $-6.40(-16.00,3.20)$ & 0.182 & $-4.48(-11.94,2.98)$ & 0.226 \\
\hline Alcohol intake (yes vs. no) & $0.03(-6.08,6.14)$ & 0.992 & $3.30(-6.76,13.35)$ & 0.505 & $-1.80(-15.46,11.86)$ & 0.787 \\
\hline Physical activity(yes vs. no) & $2.33(-5.61,10.27)$ & 0.560 & $-4.87(-17.63,7.90)$ & 0.439 & $-4.18(-12.54,4.19)$ & 0.311 \\
\hline Having chronic diseases (yes vs. no) & $-6.60(-16.82,3.62)$ & 0.202 & $-0.41(-14.42,13.61)$ & 0.952 & $-7.36(-24.88,10.15)$ & 0.392 \\
\hline BDI score & $-1.00(-1.59,-0.41)$ & 0.001 & $-1.54(-2.36,-0.73)$ & 0.001 & $-1.17(-2.22,-0.11)$ & 0.032 \\
\hline \multicolumn{7}{|l|}{ Imputed dataset } \\
\hline Age & $-0.23(-1.25,0.79)$ & 0.662 & $-1.03(-3.13,1.07)$ & 0.335 & $-0.70(-3.99,2.59)$ & 0.676 \\
\hline Gender (male vs. female) & $0.26(-3.67,4.20)$ & 0.895 & $0.31(-5.65,6.28)$ & 0.918 & $-2.37(-9.67,4.92)$ & 0.523 \\
\hline $\begin{array}{l}\text { Living arrangements } \\
\text { (dormitory vs. other) }\end{array}$ & $1.93(-2.39,6.25)$ & 0.381 & $1.16(-7.35,9.67)$ & 0.789 & $-1.03(-8.04,5.99)$ & 0.774 \\
\hline Household monthly income & $0.07(-2.02,2.15)$ & 0.949 & $-0.57(-3.69,2.53)$ & 0.715 & $-1.05(-5.15,3.05)$ & 0.613 \\
\hline Type of school (medicine vs. other) & $-3.27(-7.68,1.13)$ & 0.145 & $4.11(-2.11,10.34)$ & 0.195 & $-1.15(-8.75,6.46)$ & 0.766 \\
\hline Grade point average & $1.43(-0.89,3.75)$ & 0.227 & $-1.94(-5.57,1.70)$ & 0.296 & $-1.96(-8.23,4.30)$ & 0.537 \\
\hline Smoking (yes vs. no) & $-0.60(-5.44,4.25)$ & 0.809 & $-0.46(-7.15,6.22)$ & 0.892 & $-2.23(-10.25,5.79)$ & 0.585 \\
\hline Alcohol intake (yes vs. no) & $-0.66(-4.64,3.32)$ & 0.745 & $1.37(-6.04,8.79)$ & 0.716 & $-0.10(-10.57,10.37)$ & 0.984 \\
\hline Physical activity (yes vs. no) & $1.46(-4.53,7.46)$ & 0.631 & $-3.32(-13.65,7.02)$ & 0.527 & $-0.11(-8.40,8.18)$ & 0.979 \\
\hline Having chronic diseases (yes vs. no) & $-5.24(-11.75,1.27)$ & 0.115 & $-4.18(-14.81,6.44)$ & 0.438 & $-0.51(-16.04,15.03)$ & 0.949 \\
\hline BDI score & $-1.05(-1.44,-0.66)$ & 0.001 & $-1.35(-1.99,-0.71)$ & 0.001 & $-1.45(-2.57,-0.33)$ & 0.012 \\
\hline
\end{tabular}

BDI=Beck Depression Inventory; B=Unstandardized coefficient; $\mathrm{CI}=$ Confidence interval; P=Probability.

Kosovo $\mathrm{B}=-2.14,95 \% \mathrm{CI}:-2.82,-1.46, \mathrm{P}=0.001$; students from southern enclaves $\mathrm{B}=-2.43,95 \% \mathrm{CI}$ : $-3.53,-1.33, \mathrm{P}=0.001$ ) (Table 4$)$. In the non-imputed dataset of students from Serbia, higher grade point average was associated with better MCS $(B=$ 4.86, 95\%CI: 1.83, 7.90, $\mathrm{P}=0.002$ ) (Table 4).

\section{Discussion}

Our study suggests that HRQoL of students studying in North Kosovo does not differ according to the region of students' origin (Republic of Serbia vs. North
Kosovo vs. southern enclaves). Our hypothesis was, thus, rejected. This study found that the university student population is overall healthy, fit and resilient despite the fact that they are studying in a region with the ongoing ethnic tensions and unstable political situation. It is probable that students from the southern enclaves have stronger social ties compared with their peers in the northern Kosovo province. Also, their mobility is not restricted in Kosovska Mitrovica compared to their life at home in the enclaves. Moreover, during studies, it is expected that they participate in social events, which likely contribute to their well- 


\begin{tabular}{|c|c|c|c|c|c|c|}
\hline \multirow{3}{*}{ Variable } & \multicolumn{6}{|l|}{ Region of origin } \\
\hline & \multicolumn{2}{|l|}{ Serbia } & \multicolumn{2}{|l|}{ North Kosovo } & \multicolumn{2}{|l|}{ Southern enclaves } \\
\hline & $\mathrm{B}(95 \% \mathrm{CI})$ & $\mathrm{P}$ & B $(95 \% \mathrm{CI})$ & $\mathrm{P}$ & B $(95 \%$ CI $)$ & $\mathrm{P}$ \\
\hline \multicolumn{7}{|l|}{ Non-imputed set } \\
\hline Age & $0.90(-0.54,2.35)$ & 0.217 & $-1.38(-4.83,2.08)$ & 0.419 & $0.31(-4.81,5.42)$ & 0.902 \\
\hline Gender (male vs. female) & $1.21(-4.23,6.66)$ & 0.658 & $-0.12(-10.30,10.06)$ & 0.981 & $-10.32(-21.01,0.38)$ & 0.058 \\
\hline $\begin{array}{l}\text { Living arrangements } \\
\text { (dormitory vs. other) }\end{array}$ & $0.30(-5.49,6.09)$ & 0.919 & $7.89(-8.41,24.20)$ & 0.328 & $-8.33(-17.55,0.87)$ & 0.074 \\
\hline Household monthly income & $-0.141(-2.86,2.58)$ & 0.918 & $2.02(-2.52,6.57)$ & 0.367 & $0.06(-6.12,6.24)$ & 0.984 \\
\hline Type of school (medicine vs. other) & $-3.91(-10.12,2.31)$ & 0.214 & $6.65(-4.77,18.08)$ & 0.241 & $-4.83(-14.62,4.97)$ & 0.317 \\
\hline Grade point average & $4.86(1.83,7.90)$ & 0.002 & $-2.66(-8.50,3.17)$ & 0.355 & $1.83(-8.76,12.43)$ & 0.723 \\
\hline Smoking (yes vs. no) & $-4.45(-11.65,2.75)$ & 0.221 & $-5.84(-17.74,6.05)$ & 0.321 & $0.47(-9.93,10.86)$ & 0.926 \\
\hline Alcohol intake (yes vs. no) & $-0.39(-6.22,5.45)$ & 0.895 & $-7.48(-19.93,4.98)$ & 0.227 & $8.71(-10.32,27.73)$ & 0.352 \\
\hline Physical activity (yes vs. no) & $6.22(-1.37,13.81)$ & 0.107 & $-1.40(-17.21,14.42)$ & 0.857 & $-3.73(-15.38,7.92)$ & 0.513 \\
\hline Having chronic diseases (yes vs. no) & $-7.84(-17.61,1.93)$ & 0.114 & $9.04(-8.32,26.41)$ & 0.293 & $1.74(-22.66,26.13)$ & 0.884 \\
\hline BDI score & $-1.47(-2.03,-0.91)$ & 0.001 & $-2.30(-3.30,-1.29)$ & 0.001 & $-2.65(-4.12,-1.17)$ & 0.001 \\
\hline \multicolumn{7}{|l|}{ Imputed dataset } \\
\hline Age & $-0.57(-1.78,0.65)$ & 0.359 & $-0.88(-3.23,1.47)$ & 0.461 & $-0.34(-3.92,3.24)$ & 0.853 \\
\hline Gender (male vs. female) & $1.58(-3.08,6.25)$ & 0.506 & $-1.94(-8.50,4.62)$ & 0.561 & $-4.63(-12.33,3.06)$ & 0.238 \\
\hline $\begin{array}{l}\text { Living arrangements (dormitory vs. } \\
\text { other) }\end{array}$ & $2.89(-1.97,7.75)$ & 0.244 & $3.33(-5.74,12.40)$ & 0.471 & $-4.00(-11.48,3.48)$ & 0.294 \\
\hline Household monthly income & $-0.23(-2.71,2.25)$ & 0.855 & $0.91(-2.35,4.17)$ & 0.583 & $-0.69(-5.37,4.00)$ & 0.772 \\
\hline Type of school (medicine vs. other) & $-2.06(-7.04,2.91)$ & 0.416 & $4.12(-2.91,11.14)$ & 0.250 & $-4.50(-12.39,3.39)$ & 0.263 \\
\hline Grade point average & $1.68(-1.08,4.47)$ & 0.235 & $-2.55(-6.50,1.40)$ & 0.206 & $0.13(-6.04,6.29)$ & 0.968 \\
\hline Smoking (yes vs. no) & $-0.56(-6.34,5.21)$ & 0.848 & $-0.75(-8.15,6.66)$ & 0.843 & $1.58(-7.00,10.17)$ & 0.717 \\
\hline Alcohol intake (yes vs. no) & $-1.55(-6.46,3.35)$ & 0.535 & $-3.81(-12.38,4.75)$ & 0.381 & $1.72(-9.79,13.23)$ & 0.768 \\
\hline Physical activity (yes vs. no) & $2.67(-4.17,9.51)$ & 0.443 & $-1.64(-13.48,10.20)$ & 0.784 & $-2.12(-10.69,6.44)$ & 0.626 \\
\hline Having chronic diseases (yes vs. no) & $-1.99(-9.76,5.79)$ & 0.616 & $2.41(-8.31,13.14)$ & 0.658 & $-1.16(-16.48,14.17)$ & 0.882 \\
\hline BDI score & $-1.59(-2.07,-1.11)$ & 0.001 & $-2.14(-2.82,-1.46)$ & 0.001 & $-2.43(-3.53,-1.33)$ & 0.001 \\
\hline
\end{tabular}

BDI=Beck Depression Inventory; B=Unstandardized coefficient; $\mathrm{CI}=$ confidence interval; P=Pprobability.

being, feelings of freedom, independence and emancipation. Costs of living, such as rent, electricity, water and transportation, as well as personal expenses, are considerably lower in North Kosovo compared to those in the Republic of Serbia, which probably results in perception of higher level of comfort. All of these features combined could have influenced the stability and balance of daily life among students in North Kosovo.

In terms of physical and mental health, as measured by the PCS and MCS, our findings are consistent with those observed among students in the
Republic of Serbia (6) as well as worldwide (21, 22). Higher scores for domains of physical health reported by the students in our study suggest that they were largely active, had enough energy and good working capacity. Also, the students did not report any particular discomfort. Physical Composite Score, however, was on average higher than Mental Composite Score. In particular, the lowest reported score was observed in the domain of $\mathrm{Vi}$ tality, which contributes to the MCS. Low scores in the domain of Vitality have been closely linked to poor stress management among university stu- 
dents (23). Overall, lower Mental Composite Score among University students suggests a lack of positive feelings about future, life satisfaction, meaning and hope, highlighting that the stress management at this period of life should be prioritized and promoted in order to support students' mental wellbeing.

Our research indicates that scores in some domains of the SF-36, such as Bodily Pain, General Health, Social Functioning and Mental Health, differ between males and females, with poorer scores observed among the females. These findings are in line with those previously observed $(24,25)$. Overall, females seem to report more health problems compared with males. For example, headaches, back pain or neck/shoulder pain are more frequently reported by females (24). Females also appear to perceive greater psychological burden, because fatigue, depression and anxiety are more frequently reported among females compared to males (25). A previous study suggested that female students use health care services more often (25). The observed differences between males and females in our study could be explained by different social roles. In patriarchal societies, such as the northern Kosovo province, females are expected to take on the domestic work in the household. This, along with the academic demands, can put additional pressure on women, who expectedly try to balance their presumed social role and their personal endeavors, such as obtaining a graduate degree.

Consistent with the previous report among students in the Republic of Serbia (6), medical students generally had lower scores in all domains of the SF36 compared to students from other schools. Several studies suggested that medical students endure higher levels of stress (26), burnout (9), anxiety and depression (27) due to the demanding working hours and competitive academic environment. The classes in health sciences may also include nightshifts, which interfere with circadian functioning, particularly when people are not routinely practicing work in shifts. The extent of curricula, which is expectedly larger than in other branches, may play a role in rising anxiety, especially during exam sessions. These factors can potentially explain why students in health sciences perceive their HRQoL as worse compared to students in other branches.

The analysis of the HRQoL according to living arrangement showed that students who lived at home (with parents) had better Physical Composite Score compared to students who lived alone in rented apartments or in other places (with any family, bed-and-board, hotel), which has also been observed in Iran (28). This may be because the students who live with their parents might have more time to pursue extra-curricular activities, as parents typically tend to take care of daily duties in the household. Interestingly, students from the Republic of Serbia who lived at home with parents had better Mental Composite Score (6). By contrast, some studies worldwide reported that students living with their parents had lower self-perceived health (30). These contrasting results may be related to cultural differences, as in some cultures, people function better in close-knit communities where adult children who live with their parents are not assigned as many duties in the household, compared to when adults run their own households.

Higher BDI was consistently associated with poorer physical and mental health among students studying in the northern Kosovo province. Previous studies indicated that, overall, depression accounts for one of the most common health problems among college students $(22,27)$. Presence of depressive symptoms has been observed as a mediator between smoking and poorer HRQoL (20). Presence of depression among students may also contribute to other behaviors that might be detrimental for physical and mental health such as smoking, particularly in conflict settings (30). Screening for depression as a part of students' compulsory health checks could be helpful in early detection of depressive symptoms.

This study has some limitations. Information bias should be considered because data on smoking, alcohol consumption and physical activity were self-reported. Also, a considerable proportion of students (38.5\%) did not report their region of origin. Because of the complex socio-political cir- 
cumstances, some students feel reluctant to report their region of origin, regardless of anonymity of research. Although we remedied this issue by using the procedure of multiple imputation, we cannot be entirely certain that the imputed dataset reflect the actual distribution of students' region of origin. Due to the cross-sectional study design, causality inference is limited. Therefore, the associations observed in our study should be carefully interpreted.

\section{Conclusion}

In conclusion, the results of our study suggest that the HRQOL among Serbian students who study in North Kosovo does not differ according to the region of origin. Worse depressive symptoms were consistently associated with poorer physical and mental health among students from Serbia, North Kosovo and southern enclaves. Bearing in mind the aforementioned findings, students' health care services should focus on prevention, early recognition and treatment of depression. Screening for depression in student health care service might be a good approach to detect depressive symptoms early on.

Acknowledgement: No funding was received to conduct this study. TG, GM, DKT and TP received grant from Ministry of Education, Science and Technological Development of the Republic of Serbia (no. 175087) for other projects.

Authors' Contributions: Conception and design: MM, TG, MP, JS, NM, GM, DKT and TP; Acquisition, analysis and interpretation of data: MM, TG, DKT and TP; Drafting the article: MM and TG; Revising it critically for important intellectual content: MP, JS, NM, GM, DKT and TP; Approved final version of the manuscript: MM, TG, MP, JS, NM, GM, DKT and TP.

Conflict of Interests: The authors declare that that do not have conflict of interest.

\section{References}

1. Felix JF, Voortman T, van den Hooven EH, Sajjad A, Leermakers ET, Tharner A et al. Health in children: a conceptual framework for use in healthy ageing research. Maturitas. 2014;77(1):47-51.

2. Chan GC, Koh D. Understanding the psychosocial and physical work environment in a Singapore medical school. Singapore Med J. 2007;48:166-71.
3. Divaris K. The academic environment: The students' perspective. Eur J Dent Educ. 2008;12:120-30.

4. Stewart-Brown S, Evans J, Patterson J, Petersen S, Doll $\mathrm{H}$, Balding $\mathrm{J}$ et al. The health of students in institutes of higher education: an important and neglected public health problem? J Pub Health Med. 2000;22(4):492-9.

5. Misra R, McKean M, West S, Hibbert LJ, Brennan SL, Ross EE et al. Academic stress of college students: Comparison of student and faculty perception. College Stud J. 2000;34(2):236-45.

6. Pekmezovic T, Popovic A, Tepavcevic DK, Gazibara T, Paunic M. Factors associated with health-related quality of life among Belgrade University students. Qual Life Res. 2010;20(3):39-7.

7. Zhang Y, Qu B, Lun S, Wang D, Guo Y, Liu J. Quality of Life of Medical Students in China: A Study Using the WHOQOL-BREF. PLoS One. 2012;7(11):e49714.

8. Shareef MA, AlAmodi AA, Al-Khateeb AA, Abudan Z, Alkhani MA, Zebian SI et al. The interplay between academic performance and quality of life among preclinical students. BMC Med Educ. 2015;15:193.

9. Daniel P, Valéria de Q. Influence of burnout and sleep difficulties on the quality of life among medical students. SpringerPlus. 2015;4:676.

10. United Nations Interim Administration Mission in Kosovo (UNMIK). 2017. [cited 2017 September 5] Available from: http://www.un.org/en/peacekeeping/missions/unmik/

11. Association of Serbian Municipalities (In Serbian). 2017. [cited 2017 September 52017 ] Available from: http://www. wikiwand.com/sh/Zajednica_srpskih_op\%C5\%A1 tina

12. Office for Kosovo and Metohija Government of the Republic of Serbia. 2017 [cited 2017 September 5]. Available from: http://www.kim.gov.rs/eng/index.php

13. Organization for Security and Cooperation in Europe Mission in Kosovo. Kosovo Communities Profiles. 2010 [cited 2017 September 5]. Available from: https://www. osce.org $/$ kosovo/75450?download=true

14. Luta X, Dræbel T. Kosovo-Serbs' experiences of seeking healthcare in a post-conflict and ethnically segregated health system. Int J Public Health. 2013;58(3):377-83.

15. Mirković M, Simić S, Trajković G. Assessment of mental health in adults of the northern part of the city of Kosovska Mitrovica. Vojnosanit Pregl. 2012;69(9):747-52.

16. University of Priština temporarily settled in Kosovska Mitrovica. 2017. [cited 2017 September 15] Available from: http://www.pr.ac.rs

17. SF-36 Health survey (original version) language recalls. 2017 [cited 2017 September 15]. Available from http:// www.qualitymetric.com 
18. Beck AT, Beck RW. Screening depressed patients in family practice: A rapid technique. Postgrad Med. 1972;52:81-5.

19. Spratt M, Carpenter J, Sterne JA, Carlin JB, Heron J, Henderson $\mathrm{J}$ et al. Strategies for multiple imputation in longitudinal studies. Am J Epidemiol. 2010;172:478-87.

20. Milic M, Gazibara T, Pekmezovic T, Kisic Teparcevic D, Maric G, Popovic A. Tobacco smoking and health-related quality of life among university students: Mediating effect of depression. PLoS One. 2020;15(1):e0227042.

21. Fallahzadeh H, Hamed Mirzaei BH. Health Related Quality of life and Associated Factors among Iranian University Students. J Commun Health Res. 2012;1(2):122-30.

22. Arslan G, Ayranci U, Unsal A, Arslantas D. Prevalence of depression, its correlates among students, and its effect on health-related quality of life in a Turkish university. Ups J Med Sci. 2009;114:170-7.

23. Alborzkouh P, Nabati M, Zainali M, Abed Y, Shahgholy Ghahfarokhi F. A review of the effectiveness of stress management skills training on academic vitality and psychological well-being of college students. J Med Life. 2015;8(Spec Iss 4):39-44.

24. El Ansari W, Stock C, UK Student Health Group. Feeling healthy? A survey of physical and psychological wellbeing of students from seven universities in the UK. Int J Environ Res Public Health. 2011;8(5):1308-23.

25. Vaez M, Laflamme L. First-year university students' health status and socio-demographic determinants of their selfrated health. Work. 2002;19(1):71-80.

26. Bhandari P. Stress and health related quality of life of Nepalese students studying in South Korea: a cross sectional study. Health Qual Life Outcomes. 2012;13:10-26.

27. Jadoon NA, Yaqoob R, Raza A, Shehzad MA, Zeshan SC. Anxiety and depression among medical students:a crosssectional study. J Pak Med Assoc. 2010;60(8):699-702.

28. Jamali A, Tofangchiha S, Jamali R, Nedjat S, Jan D, Narimani A et al. Medical students' health-related quality of life: roles of social and behavioural factors. Med Educ. 2013;47(10):1001-12.

29. Boot CR, Rietmeijer CB, Vonk P, Meijman FJ. Perceived health profiles of Dutch university students living with their parents, alone or with peers. Int J Adolesc Med Health. 2009;21(1):41-9.

30. Idris A, Al Saadi T, Turk T, Alkhatib M, Zakaria M, Sawaf $\mathrm{B}$ et al. Smoking behaviour and patterns among university students during the Syrian crisis. East Mediterr Health J. 2018;24(2):154-60. 


\section{Supplemental Material}

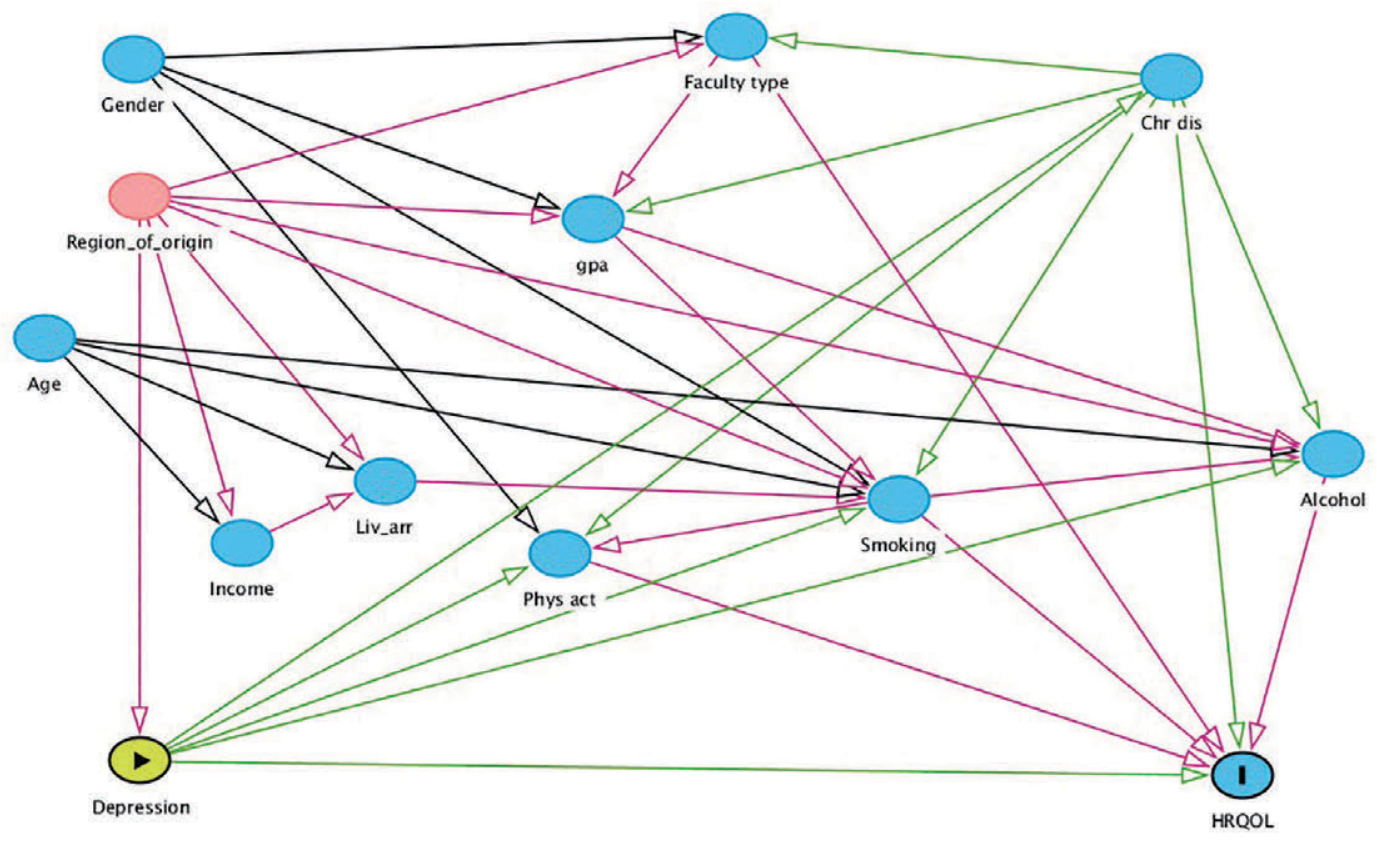

Fig. S1. Directed Acyclic Graph (DAG): Relationship between the Variables, Moderators and Mediators of the Association between Depression and Health-related Quality of Life among Students.

\section{Table S1. Gender-specific Mean Scores of the Sf-36 Scales}

\begin{tabular}{lllll}
\hline \multirow{2}{*}{ Scales of SF-36 } & Both genders & Male & Female \\
\cline { 2 - 4 } & Mean \pm SD & Mean \pm SD & Mean \pm SD & 0.067 \\
\hline Physical functioning & $92.8 \pm 17.7$ & $93.9 \pm 16.3$ & $91.9 \pm 18.7$ & 0.237 \\
\hline Role physical & $85.9 \pm 26.2$ & $84.7 \pm 26.9$ & $87.0 \pm 25.5$ & 0.002 \\
\hline Pain & $85.8 \pm 17.8$ & $88.0 \pm 17.4$ & $83.8 \pm 17.9$ & 0.001 \\
\hline General health & $74.4 \pm 17.0$ & $77.1 \pm 16.8$ & $72.0 \pm 16.9$ & 0.075 \\
\hline Vitality & $68.8 \pm 20.3$ & $70.6 \pm 19.6$ & $67.2 \pm 20.7$ & 0.014 \\
\hline Social functioning & $81.3 \pm 22.2$ & $83.3 \pm 22.3$ & $79.6 \pm 22.0$ & 0.148 \\
\hline Role emotional & $79.2 \pm 35.2$ & $81.8 \pm 33.2$ & $77.1 \pm 36.7$ & 0.022 \\
\hline Mental health & $74.2 \pm 19.8$ & $76.3 \pm 18.9$ & $72.4 \pm 20.4$ & 0.016 \\
\hline Physical composite score & $81.5 \pm 13.6$ & $82.9 \pm 13.3$ & $80.4 \pm 13.8$ & 0.005 \\
\hline Mental composite score & $75.6 \pm 17.3$ & $77.8 \pm 16.7$ & $73.7 \pm 17.6$ & 0.007 \\
\hline Total score & $80.3 \pm 15.2$ & $82.0 \pm 14.9$ & $78.9 \pm 15.3$ & \\
\hline
\end{tabular}

*Standard deviation; P-value for ANOVA. 
Table S2. Scores of the Sf-36 Scales according to Current Living Arrangement

\begin{tabular}{|c|c|c|c|c|c|}
\hline \multirow[t]{2}{*}{ Scales of SF-36 } & Home (with parents) & Student's dormitory & Alone (in rented apartment) & $\begin{array}{l}\text { Other (with relatives, } \\
\text { bed-and-board, hotel) }\end{array}$ & \multirow[t]{2}{*}{$P$ value } \\
\hline & Mean \pm SD & Mean \pm SD & Mean \pm SD & Mean \pm SD & \\
\hline Physical functioning & $93.3 \pm 17.2$ & $92.7 \pm 18.3$ & $92.9 \pm 17.1$ & $89.3 \pm 21.5$ & 0.597 \\
\hline Role physical & $90.4 \pm 21.3$ & $87.3 \pm 24.8$ & $82.1 \pm 29.6$ & $71.7 \pm 33.9$ & $0.002^{\dagger}$ \\
\hline Pain & $88.2 \pm 16.6$ & $87.4 \pm 16.4$ & $83.0 \pm 19.3$ & $73.5 \pm 19.3$ & $0.002^{\ddagger}$ \\
\hline General health & $76.2 \pm 15.4$ & $75.6 \pm 16.9$ & $72.7 \pm 17.9$ & $62.0 \pm 16.6$ & $0.008^{\ddagger}$ \\
\hline Vitality & $71.0 \pm 20.4$ & $70.5 \pm 19.5$ & $65.9 \pm 19.9$ & $58.0 \pm 26.3$ & $0.009^{\S}$ \\
\hline Social functioning & $82.9 \pm 20.9$ & $81.2 \pm 21.9$ & $80.9 \pm 23.0$ & $73.3 \pm 28.3$ & 0.609 \\
\hline Role emotional & $81.7 \pm 33.1$ & $81.0 \pm 34.9$ & $76.9 \pm 36.5$ & $62.2 \pm 39.6$ & 0.100 \\
\hline Mental health & $75.3 \pm 20.6$ & $75.6 \pm 18.8$ & $72.4 \pm 19.9$ & $67.7 \pm 21.8$ & 0.185 \\
\hline Physical composite score & $83.8 \pm 11.5$ & $82.7 \pm 13.1$ & $79.3 \pm 14.7$ & $70.9 \pm 18.1$ & $0.001^{\| \prime}$ \\
\hline Mental composite score & $77.4 \pm 16.7$ & $76.8 \pm 16.6$ & $73.7 \pm 17.8$ & $64.7 \pm 21.6$ & 0.028 \\
\hline Total score & $82.4 \pm 13.7$ & $81.4 \pm 14.6$ & $78.3 \pm 16.1$ & $69.7 \pm 19.7$ & $0.007^{9}$ \\
\hline
\end{tabular}

"Standard deviation; P-value for ANOVA; ${ }^{\dagger}$ Home vs. rented apartments and other; ${ }^{\ddagger}$ Home and dormitory vs. Other; $\$$ Home vs. rented apartment; "Home vs. rented apartment and Other as well as dormitory vs. Other; 'Home vs. Other.

\section{Table S3. Scores of the Sf-36 Scales according to Type of Faculty}

\begin{tabular}{llllll}
\hline \multirow{2}{*}{ Scales of SF-36 } & $\begin{array}{l}\text { Social sciences and } \\
\text { humanities }\end{array}$ & Health sciences & $\begin{array}{l}\text { Natural sciences and } \\
\text { mathematics }\end{array}$ & $\begin{array}{l}\text { Technology and } \\
\text { Engineering } \\
\text { sciences }\end{array}$ & P value \\
\cline { 2 - 5 } & Mean \pm SD $^{*}$ & Mean \pm SD $^{*}$ & Mean \pm SD $^{*}$ & Mean \pm SD $^{*}$ \\
\hline Physical functioning & $88.1 \pm 24.2$ & $95.0 \pm 12.8$ & $95.0 \pm 7.6$ & $93.8 \pm 15.4$ & 0.129 \\
\hline Role physical & $87.6 \pm 26.8$ & $85.0 \pm 26.7$ & $78.6 \pm 35.2$ & $86.8 \pm 23.0$ & 0.333 \\
\hline Pain & $86.4 \pm 17.4$ & $83.6 \pm 18.9$ & $84.3 \pm 16.0$ & $89.6 \pm 15.5$ & $0.025^{\dagger}$ \\
\hline General health & $73.6 \pm 16.2$ & $72.8 \pm 17.5$ & $72.9 \pm 21.0$ & $78.9 \pm 15.7$ & $0.006^{\ddagger}$ \\
\hline Vitality & $70.1 \pm 20.9$ & $65.1 \pm 20.6$ & $72.5 \pm 14.4$ & $74.2 \pm 18.0$ & $0.001^{5}$ \\
\hline Social functioning & $78.8 \pm 22.7$ & $81.6 \pm 23.6$ & $83.9 \pm 15.8$ & $83.4 \pm 19.1$ & 0.374 \\
\hline Role emotional & $82.1 \pm 33.2$ & $76.1 \pm 38.0$ & $81.0 \pm 31.2$ & $79.6 \pm 16.5$ & 0.517 \\
\hline Mental health & $72.2 \pm 19.8$ & $72.2 \pm 19.8$ & $84.0 \pm 13.1$ & $84.6 \pm 10.6$ & $0.001^{\ddagger}$ \\
\hline Physical composite score & $81.2 \pm 14.7$ & $80.3 \pm 14.0$ & $80.6 \pm 15.2$ & $79.6 \pm 14.5$ & 0.072 \\
\hline Mental composite score & $75.3 \pm 17.4$ & $73.5 \pm 18.5$ & $78.8 \pm 13.7$ & $83.5 \pm 12.0$ & 0.054 \\
\hline Total score & $79.9 \pm 16.1$ & $78.9 \pm 16.0$ & $81.5 \pm 13.6$ & 0.188 \\
\hline
\end{tabular}

"Standard deviation; P-value for ANOVA; ${ }^{\dagger}$ Health vs. Technology and engineering; ${ }^{\ddagger}$ Health and Social sciences and humanities vs. Technology and engineering; ${ }^{\varsigma} \mathrm{Health}$ vs. Social sciences and humanities and Technology and engineering. 